\title{
Deuxième intervention tarifaire: nombreuses questions en suspens
}

\section{Urs Stoffel}

Dr méd., membre du Comité central de la FMH, responsable du département Médecine et tarifs ambulatoires

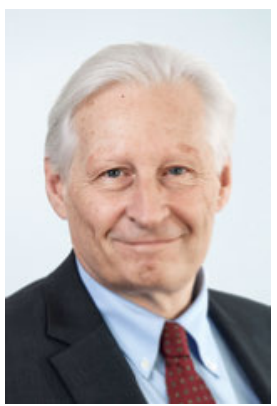

Le 16 août 2017, le Conseil fédéral a tranché. Mais alors que l'on s'attendait à ce qu'il publie l'ordonnance définitive en vue de son intervention dans le TARMED, celle-ci ne devrait être divulguée qu'à l'automne.

Dans son communiqué de presse, le Conseil fédéral qualifie de décision de principe son intervention dans le tarif au $1^{\text {er }}$ janvier 2018. Comme la convention-cadre TARMED a été dénoncée au 31 décembre 2017, le Conseil fédéral imposera son propre tarif à partir du $1^{\text {er }}$ janvier 2018. Les détails de cette structure tarifaire (TARMED 1.09_BR) ne sont pas encore connus.

Hormis cette décision de principe, le Conseil fédéral a publié une fiche d'information présentant les principaux changements apportés à la version mise en consultation.

Le Conseil fédéral a manifestement pris conscience que sa proposition mise en consultation risquait de mettre en danger les soins ambulatoires et la qualité élevée du système de santé de notre pays. Les critiques émises par les sociétés médicales ont en partie été entendues; il n'en demeure pas moins que cette intervention du Conseil fédéral va perturber la prise en charge médicale ambulatoire au lieu de la renforcer comme le souhaite le milieu politique. Un mauvais signal dans la mauvaise direction, donc.

\section{Le Conseil fédéral a en partie corrigé sa} proposition, mais son intervention dans le tarif reste inappropriée.

Le 18 août 2017, les présidents de Médecins de famille et de l'enfance Suisse (mfe), des médecins avec activité chirurgicale et invasive ( $\mathrm{fmCh}$ ) et des médecins psychiatres-psychothérapeutes (FMPP) ont pour la première fois pris position ensemble aux côtés de la délégation de la FMH sur la décision du Conseil fédéral et sur l'intervention tarifaire. Une conférence de presse commune historique, qualifiée par le président de la FMPP de "2 $2^{\mathrm{e}}$ miracle de Berne». Malgré quelques adaptations pour les enfants de moins de six ans, les personnes âgées, les personnes atteintes de troubles psychiques, les urgences et certaines limitations, l'intervention tarifaire reste inappropriée. En effet, les prestations fournies aux enfants de plus de six ans ou encore la suppression de prestations à l'acte chez les dermatologues par exemple, tout comme de nombreuses autres mesures ne répondent pas aux règles de l'économie d'entreprise. Des lacunes que les présidents des organisations faîtières et la FMH ont illustrées à l'aide d'exemples lors de la conférence de presse.

On ne peut pas défendre le principe de «l'ambulatoire avant l'hospitalier" tout en affaiblissant la médecine ambulatoire et en refusant de participer au financement de l'augmentation - voulue - des prestations ambulatoires sur le dos des payeurs de primes.

\section{Le corps médical serre les rangs pour une} révision globale.

Les représentants du corps médical se sont tous accordés à dire que nous avons besoin d'un tarif ambulatoire fort, approprié et développé en partenariat, qui réponde aux critères de l'économie d'entreprise, pour être en mesure de répondre à la volonté politique de la Confédération et des cantons de privilégier «l'ambulatoire avant l'hospitalier".

Les représentants des organisations faîtières et la FMH ont profité de la conférence de presse pour souligner clairement et à l'unisson qu'une révision globale appropriée du tarif ambulatoire TARMED est, pour le corps médical, l'unique voie à suivre pour renforcer la médecine ambulatoire de la manière la plus avantageuse et pour se préparer efficacement aux défis à venir. Fortes de ce constat, la FMH et les organisations représentées à la Chambre médicale poursuivent ensemble la révision globale du TARMED dans le cadre du projet TARCO dans le but de remettre le tarif révisé pour approbation au Conseil fédéral en juin 2018 avec tous les partenaires tarifaires. 